\title{
COMMUNICATION
}

\section{Carcinome odontogène à cellules claires de la mandibule, difficultés du diagnostic}

\section{Ordioni U', Benat G², Hadj Saïd $\mathrm{M}^{1,3}$, Gallucci $\mathrm{A}^{3}$, Massereau $\mathrm{E}^{1,3}$, Catherine $\mathrm{JH}^{1,4}$}

1 - Service d'Odontologie, Assistance Publique Hôpitaux de Marseille, Hôpital de la Timone, 264 rue Saint Pierre, 13005 Marseille

2 - Service d'Anatomie et Cytologie Pathologiques, Institut Universitaire du Cancer de Toulouse-Oncopole, 1 avenue Irène Joliot-Curie, 31059 Toulouse

3 - Service de Stomatologie et Chirurgie Maxillo-Faciale, Assistance Publique Hôpitaux de Marseille, Hôpital de la Conception, 147 boulevard Baille, 13005 Marseille

4 - UMR 7268 ADES, Aix-Marseille Université/EFS/CNRS, Faculté de Médecine- secteur Nord, Bd Pierre Dramard, 13344, Marseille Cedex 15

Thèmes : Chirurgie, Médecine, Anatomopathologie, Cancérologie

Introduction

Le carcinome odontogène à cellules claires (COCC) est une tumeur exeptionnelle décrite par Hansen et al. en 1985. D'abord appelée tumeur odontogène à cellules claires, elle était considérée comme une lésion bénigne à agressivité locale dans la classification des tumeurs de l'OMS en 1992. Le terme de COCC apparaît en 2005 dans la révision de cette classification où elle est reconnue comme une tumeur maligne (Barnes, 2005). Avec 116 cas recensés dans la littérature depuis sa description, cette entité présente des difficultés diagnostiques majeures (Loyola, 2015).

\section{Observation}

Un patient de 64 ans sans antécédents généraux, était adressé dans le service de chirurgie orale du centre hospitalier universitaire de La Timone à Marseille pour la prise en charge d'une lésion intraosseuse radioclaire secteur 4 . Le patient présentait une paresthésie de l'hémilèvre droite. L'examen tomodensitométique de cette lésion mettait en évidence une lésion ostéolytique agressive, avec un envahissement osseux bicortical. L'IRM montrait un tropisme nerveux avec envahissement du nerf alvéolaire inférieur droit par la lésion. Une biopsie était réalisée, mettant en évidence une prolifération tumorale épithéliale de nature odontogène à cellules claires, agressive, dont le diagnostic était difficile. Les hypothèses diagnostiques étaient dans un premier temps une lésion métastatique d'un carcinome à cellules claires du rein, un COCC ou une tumeur squameuse odontogène. Le bilan d'extension permettait d'éliminer une lésion primitive rénale. Les lames étaient adressées à l'institut Universitaire du cancer de Toulouse. Devant l'absence de mitose, le diagnostic retenu était celui de tumeur squameuse odontogène. La prise en charge avait consisté après discussion en RCP en une exérèse large de la lésion par hémi-mandibulectomie interruptrice associée à un curage ganglionnaire et à une reconstruction par un lambeau microanastomosé de fibula. L'examen histologie de la pièce opératoire a conclu 
devant la présence d'atypies marquées, d'une activité mitotique modérée et d'une fission du gène EWSRI à un COCC.

Discussion

Ce cas clinique illustre la difficulté diagnostique des COCC. Le diagnostic différentiel se fait avec des adénocarcinomes salivaires à cellules claires, des tumeurs mélanotiques, métastatique rénale ou d'autres tumeurs épithéliales odontogènes telles que l'améloblastome ou la tumeur squameuse odontogène ou la tumeur épithéliale odontogène calcifiée (Barnes, 2005). Les examens cliniques et radiologiques orientent vers une lésion agressive. L'interprétation anatomopathologique est complexe, de nombreuses tumeurs ou métastases présentant des cellules claires. Le traitement est chirurgical, une radiothérapie post-opératoire doit être discutée en fonction des marges. Le taux de survie à 13 ans est de $21 \%$.

\section{Conclusion}

Le comportement agressif de cette tumeur qui présente un potentiel métastatique ganglionnaire et/ou pulmonaire et des récidives fréquentes impose un traitement chirurgical radical. L'immunohistochimie est une des clés du diagnostic.

Barnes L et al. WHO Classification of Tumors, Pathology and Genetics of Head and Neck Tumours. Lyon, France: IARC Press; 2005. WHO histological classification of odontogenic tumours; p. 284.

Loyola et al. Oral Surg Oral Med Oral Pathol Oral Radiol. 2015 Oct;120(4):483-96. 